\title{
Reconstructing the spatiotemporal patterns of admixture during the European Holocene using a novel genomic dating method
}

\author{
Manjusha Chintalapati $^{1 \#}$, Nick Patterson ${ }^{2 \#}$, Priya Moorjani ${ }^{1,3 \#}$ \\ ${ }^{1}$ Department of Genetics, University of California, Berkeley, CA 94620 USA \\ ${ }^{2}$ Broad Institute of Harvard and MIT, Cambridge, MA, 02142, USA \\ ${ }^{3}$ Center for Computational Biology, University of California, Berkeley, CA 94620 USA
}

\begin{abstract}
Recent studies have shown that gene flow or admixture has been pervasive throughout human history. While several methods exist for dating admixture in contemporary populations, they are not suitable for sparse, low coverage data available from ancient specimens. To overcome this limitation, we developed DATES that leverages ancestry covariance patterns across the genome of a single individual to infer the timing of admixture. By performing simulations, we show that DATES provides reliable results under a range of demographic scenarios and outperforms available methods for ancient DNA applications. We apply DATES to $\sim 1,100$ ancient genomes to reconstruct gene flow events during the European Holocene. Present-day Europeans derive ancestry from three distinct groups, local Mesolithic hunter-gatherers, Anatolian farmers, and Yamnaya Steppe pastoralists. These ancestral groups were themselves admixed. By studying the formation of Anatolian farmers, we infer that the gene flow related to Iranian Neolithic farmers occurred no later than 9,600 BCE, predating agriculture in Anatolia. We estimate the early Steppe pastoralist groups genetically formed more than a millennium before the start of steppe pastoralism, providing new insights about the history of proto-Yamnaya cultures and the origin of Indo-European languages. Using ancient genomes across sixteen regions in Europe, we provide a detailed chronology of the Neolithization across Europe that occurred from $\sim 6,400-4,300$ BCE. This movement was followed by a rapid spread of steppe ancestry from $\sim 3,200-2,500$ BCE. Our analyses highlight the power of genomic dating methods to elucidate the legacy of human migrations, providing insights complementary to archaeological and linguistic evidence.
\end{abstract}

\section{Keywords}

genomic clocks, admixture, ancient DNA, European Holocene, molecular clock, migration, Neolithic, Bronze Age, Yamnaya 


\section{Significance}

40 The European continent was subject to two major migrations during the Holocene: the

41 movement of Near Eastern farmers during the Neolithic and the migration of Steppe

42 pastoralists during the Bronze Age. To understand the timing and dynamics of these

43 movements, we developed DATES that leverages ancestry covariance patterns across the

44 genome of a single individual to infer the timing of admixture. Using $\sim 1,100$ ancient genomes

45 spanning $\sim \mathbf{8 , 0 0 0}-350 \mathrm{BCE}$, we reconstruct the chronology of the formation of the ancestral

46 populations and the fine-scale details of the spread of Neolithic farming and Steppe

47 pastoralist-related ancestry to Europe. Our analysis demonstrates the power of genomic

48 dating methods to provide an independent and complementary timeline of population origins

49 and movements using genetic data. 


\section{Introduction}

Recent studies have shown that population mixture (or "admixture") is pervasive throughout human history, including mixture between the ancestors of modern humans and archaic hominins (i.e., Neanderthals and Denisovans), as well as in the history of many contemporary human groups such as African Americans, South Asians and Europeans (1, 2). Many admixed groups are formed due to population movements involving ancient migrations that pre-date historical records. The recent availability of genomic data for a large number of present-day and ancient genomes provides an unprecedented opportunity to reconstruct population events using genetic data, providing evidence complementary to linguistics and archaeology. Understanding the timing and signatures of admixture offers insights into the historical context in which the mixture occurred and enables the characterization of the evolutionary and functional impact of the gene flow.

To characterize patterns of admixture, genetic methods use the insight that the genome of an admixed individual is a mosaic of chromosomal segments inherited from distinct ancestral populations (3). Due to recombination, these ancestral segments get shuffled in each generation and become smaller and smaller over time. The length of the segments is inversely proportional to the time elapsed since the mixture $(3,4)$. Several genetic approaches-ROLLOFF (4), ALDER (5), Globetrotter (2), and Tracts (6) - have been developed that use this insight by characterizing patterns of admixture linkage disequilibrium (LD) or haplotype lengths across the genome to infer the timing of mixture. Haplotype-based methods perform chromosome painting or local ancestry inference at each locus in the genome and characterize the distribution of ancestry tract lengths to estimate the time of mixture $(2,6)$. This requires accurate phasing and inference of local ancestry, which is often difficult when the admixture events are old (as ancestry blocks become smaller over time) or when reference data from ancestral populations is unavailable. Admixture LD-based methods, on the other hand, measure the extent of the allelic correlation across markers to infer the time of admixture $(4,5)$. They do not require phased data from the target or reference populations and work reliably for dating older admixture events (>100 generations). However, they tend to be less efficient in characterizing admixture events between closely related ancestral groups.

While highly accurate for dating admixture events using data from present-day samples, current methods do not work reliably for dating admixture events using ancient genomes. Ancient DNA samples often have high rates of DNA degradation, contamination (from human and other sources) and low sequencing depth, leading to a large proportion of missing variants and uneven coverage across the genome. Additionally, most studies generate pseudo-homozygous genotype calls - consisting of a single allele call at each diploid site - that can lead to some issues in the inference. In such sparse datasets, estimating admixture LD can be noisy and biased (see Simulations below). Moreover, haplotype-based methods require phased data from both admixed and reference populations which remains challenging for ancient DNA specimens. 
An extension of admixture LD-based methods, recently introduced by Moorjani et al. (2016), leverages ancestry covariance patterns that can be measured in a single sample using low coverage data. This approach measures the allelic correlation across neighboring sites, but instead of measuring admixture LD across multiple samples, it integrates data across markers within a single diploid genome. Using a set of ascertained markers that are informative for Neanderthal ancestry (where sub-Saharan Africans are fixed for the ancestral alleles and Neanderthals have a derived allele), Moorjani et al. (2016) inferred the timing of Neanderthal gene flow in Upper Paleolithic Eurasian samples and showed the approach works accurately in ancient DNA samples (1). However, this approach is inapplicable for dating admixture events within modern human populations, as there are very few fixed differences across populations (7).

Motivated by the single sample statistic in Moorjani et al. (2016), we developed DATES (Distribution of Ancestry Tracts of Evolutionary Signals) that measures the ancestry covariance across the genome in a single admixed individual, weighted by the allele frequency difference between two ancestral populations. This method was first introduced in Narasimhan et al. (2019), where it was used to infer the date of gene flow between groups related to Ancient Ancestral South Indians, Iranian farmers, and Steppe pastoralists in ancient South and Central Asian populations (8). In this study, we evaluate the performance of DATES by performing extensive simulations for a range of demographic scenarios and compare the approach to other published genomic dating methods. We then apply DATES to infer the chronology of the genetic formation of the ancestral populations of Europeans and the spatiotemporal patterns of admixture during the European Holocene using data from 1,100 ancient DNA specimens spanning 8,000-350 BCE.

\section{Results}

\section{Overview of DATES: Model and simulations}

DATES estimates the time of admixture by measuring the weighted ancestry covariance across the genome using data from a single diploid genome and two reference populations (representing the ancestral source populations). DATES works like haplotype-based methods as it is applicable to dating admixture in a single genome and not like admixture LD-based methods, which by definition require multiple genomes to be co-analyzed; but unlike haplotype-based methods, it is more flexible as it does not require local ancestry inference. There are three main steps in DATES: we start by first learning the genome-wide ancestry proportions by performing a simple regression analysis to model the observed genotypes in an admixed individual as a linear mix of allele frequencies from the two reference populations. For each marker, we then compute the likelihood of the observed genotype in the admixed individual using the estimated ancestry proportions and allele frequencies in each reference population (this is similar in spirit to local ancestry inference). This information is, in turn, used to compute the joint likelihood for two neighboring markers to test if they derive ancestry from the same ancestral group, accounting for the probability of recombination between the two markers. Finally, we compute the covariance 
across pairs of markers located at a particular genetic distance, weighted by the allele frequency differences in the reference populations (Note S1).

Following (1), we bin the markers that occur at a similar genetic distance across the genome, rather than estimating admix ture LD for each pair of markers, and compute the covariance across increasing genetic distance between markers. The estimated covariance is expected to decay exponentially with genetic distance, and the rate of decay is informative of the time of the mixture (4). Assuming the gene flow occurred instantaneously, we infer the average date of gene flow by fitting an exponential distribution to the decay pattern (Methods). In cases where data for multiple individuals is available, we compute the likelihood by summing over all individuals. To make DATES computationally tractable, we implemented the fast Fourier transform (FFT) for calculating ancestry covariance as described in ALDER (5). This provides a speedup from $O\left(n^{2}\right)$ to $O(n \log n)$, which reduces the typical runtimes from hours to seconds (Note $\mathrm{S} 1$ ).

To assess the reliability of DATES, we performed simulations where we constructed ten admixed diploid genomes by randomly sampling haplotypes from two source populations (Note S2). Briefly, we simulated individual genomes with 20\% European and $80 \%$ African ancestry by using phased haplotypes of Northern Europeans (Utah European Americans, CEU) and west Africans (Yoruba from Nigeria, YRI) from the 1000 Genomes Project respectively (7). As reference populations in DATES, we used closely related surrogate populations of French and Yoruba respectively, from the Human Genome Diversity Panel (9). We first investigated the accuracy of DATES by varying the time of admixture between 10-300 generations. For comparison, we also applied ALDER (5) to these simulations. Both methods reliably recovered the time of admixture up to 200 generations or $\sim 5,600$ years ago, assuming a generation time of 28 years (1), though DATES was more precise than ALDER for older admixture events $(>100$ generations) (Table S2.4). Further, DATES shows accurate results even for single samples (Figure 1).

Next, we tested DATES for features such as varying admixture proportions and use of surrogate populations as reference groups. By varying of European ancestry proportion between $\sim 1-50 \%$ (the rest derived from west Africans), we observed DATES accurately estimated the timing in all cases (Figure S2.2A). However, the inferred admixture proportion was overestimated was lower admixture proportions $(<10 \%)$ (Figure S2.2B). Thus, we caution against using DATES for estimating ancestry proportions and recommend other methods based on $f$-statistics (10). Using reference populations which are divergent from true admixing source, we found that the inferred dates were accurate even when we used Khomani San instead of Yoruba as the reference population $\left(F_{S T} \sim 0.1\right)$ (Figure $\left.S 2.5\right)$. We also found that using the admixed samples themselves as one of the reference populations also works reliably as ALDER (i.e., single reference setup) (5).

An important feature of DATES is that it does not require phased data and is applicable to datasets with small sample sizes, making it in principle useful for ancient DNA applications. To test the reliability of DATES for ancient genomes, we simulated data mimicking the relevant features of ancient genomes, namely with large proportions of missing genotypes (between 10$60 \%$ ), and pseudo-homozygous genotype calls (instead of diploid genotype calls). DATES showed 
reliable results in both cases, even only a single admixed individual was available (Figure S2.7). In contrast, admixture LD-based methods require more than one sample and do not work reliably with missing data. For example, ALDER estimates were very unstable for simulations with $>40 \%$ missing data. For older dates (>100 generations), we observed slight bias even with $>10 \%$ missing genotypes (Figure S2.17). As LD calculations leverage shared patterns across samples, variable missingness of genotypes across individuals leads to substantial loss of data leading to unstable and noisy inference. This highlights a major advantage of DATES for ancient DNA studies as it provides reliable results even in sparse datasets (Note S2.5).

DATES assumes a model of instantaneous gene flow with a single pulse of mixture between two source populations. However, many human populations have a history of multiple pulses of gene flow. To test the performance of DATES for multi-way admixture events, we generated admixed individuals with ancestry from three sources (East Asians, Africans, and Europeans) where the gene flow occurred at two distinct time points (Note S2, Figure S2.10). By applying $D A T E S$ with pairs of reference populations at a time and fitting a single exponential to the ancestry covariance patterns, we observed that DATES recovered both admixture times in case of equal ancestry proportion from the three ancestral groups when the associated reference groups were used for dating (Figure S2.11). In the case of unequal admixture proportions from three ancestral groups, DATES inferred the timing of the recent admixture event in most cases, though some confounding was observed, especially when the ancestry proportion of the recent event was low (Figure S2.12). However, if the reference populations were set up to match the model of gene flow, we observed that we could reliably recover the time of the recent gene flow event. For example, there is limited confounding if the two references used in DATES include (i) the source population for the recent event and (ii) either the pooled ancestral populations contributing to the first (or earlier) event or the intermediate admixed group formed after the first event (Table S2.1). This highlights how the choice of reference populations can help to tune the method to infer the timing of specific admixture events reliably.

Finally, we explored the impact of more complex demographic events, including continuous admixture and founder events using coalescent simulations (Note S2). In the case of continuous admixture, DATES inferred an intermediate timing between the start and the end of the gene flow period, similar to other methods like ALDER and Globetrotter $(2,5)$ (Table S2.2). In the case of populations with founder events, we inferred unbiased dates of admixture in most cases except when the founder event was extreme $\left(N_{e} \sim 10\right)$ or the population had maintained a low population size $\left(N_{e}<100\right)$ until present (i.e., no recovery bottleneck) (Figure S2.13, Table S2.3). In humans, few populations have such extreme founder events, and thus, in most other cases, our inferred admixture dates should be robust to founder events (11). We note that while DATES is not a formal test of admixture, in simulations, we find that in the absence of gene flow, the method does not infer significant dates of admixture even when the target has a complex demographic 


\section{Comparison to other methods}

We assessed the reliability of DATES in real data by comparing our results with published methods: Globetrotter, ALDER, and ROLLOFF. These methods are designed for the analysis of present-day samples that typically have high-quality data with limited missing variants. In addition, Globetrotter uses phased data which is challenging for ancient DNA samples. Thus, instead of rerunning other methods, we took advantage of the published results for contemporary samples presented in Hellenthal et al., (2014) (2). Following (2), we created a merged dataset including individuals from Human Genome Diversity Panel (9), Behar et al. (2010) (12), and Henn et al. (2012) (13) (Methods). We applied DATES and ALDER to 29 target groups using the reference populations reported in Hellenthal et al. 2014 (Table S12), excluding one group where the population label was unclear. Interestingly, the majority of these groups (25/29) failed ALDER's formal test of admixture; either because the results of the single reference and two reference analyses yielded inconsistent estimates or because the target had long-range shared LD with one of the reference populations (Table S4.1). Using DATES, we inferred significant dates of admixture in 20 groups, and 14 of those were consistent with estimates based on Globetrotter. In most remaining cases, recent studies suggest the target populations may have ancestry from multiple gene flow events, either involving the same source populations or additional ancestral groups . The estimated admixture timing based on DATES, ROLLOFF, and ALDER (assuming two-way admixture regardless of the formal test results) were found to be highly concordant (Table S4.1).

Recent ancient DNA studies have shown that present-day Europeans derive ancestry from three distinct sources: (a) hunter-gatherer-related ancestry that is closely related to Mesolithic huntergatherers (HG) from Europe; (b) Anatolian farmer-related ancestry related to Neolithic farmers from the Near East and associated to the spread of farming to Europe; and (c) Steppe pastoralistrelated ancestry that is related to the Yamnaya pastoralists from Russia and Ukraine (16-19). Many open questions remain about the timing and dynamics of these population interactions, in particular related to the formation of the ancestral groups (which were themselves admixed) and their expansion across Europe. To characterize the spatial and temporal patterns of mixtures in Europe in the past 10,000 years, we used 1,096 ancient European samples from 152 groups from the publicly available Allen Ancient DNA Resource (AADR) spanning a time range of 8,000-350 events, and below, we describe the key events in chronological order focusing on three main periods.

Holocene to Mesolithic: Pre-Neolithic Europe was inhabited by hunter-gatherers until the arrival of the first farmers from the Near East $(20,21)$. There was large diversity among hunter-gatherers with four main groups - western hunter-gatherers (WHG) that were related to the Villabruna 
cluster in central Europe, eastern hunter-gatherers (EHG) from Russia and Ukraine related to the Upper Paleolithic group of Ancestral North Eurasians (ANE) ancestry, Caucasus hunter-gatherers $(\mathrm{CHG})$ from Georgia associated to the first farmers from Iran, and the GoyetQ2-cluster associated to the Magdalenian culture in Spain and Portugal (18, 22-25). Most Mesolithic HGs fall on two main clines of relatedness: one cline that extends from Scandinavia to central Europe showing variable WHG-EHG ancestry, and the other in southern Europe with WHG-GoyetQ2 ancestry (23). This ancestry is already present in the 17,000 BCE El Mirón individual from Spain, suggesting that the GoyetQ2-related gene flow occurred well before the Holocene. However, the WHG-EHG cline was formed more recently during the Mesolithic period, though the precise timing of the spread of EHG ancestry remains less well understood.

To characterize the formation of the WHG-EHG cline, we used genomic data from 16 ancient HG groups ( $n=101)$ with estimated ages of $\sim 7,500-3,600$ BCE. We first verified the ancestry of each HG group using $q p A d m$ that compares the allele frequency correlations between the target and a set of source populations to formally test the model of admixture and then infer the ancestry proportions for the best fitted model (16). For each target population, we chose the most parsimonious model, i.e., fitting the data with the minimum number of source populations. Consistent with previous studies, our $q p A d m$ analysis showed that most HGs from Scandinavia, the Baltic Sea region, and central Europe could be modeled as a two-way mixture of WHG and EHG-related ancestry (Table S5.1, Note S5). To confirm that the target populations do not harbor Anatolian farmer-related ancestry (that could lead to some confounding in estimated admixture dates), we applied $D$-statistics of the form $D$ (Mbuti, target, WHG, Anatolian farmers) where target $=$ Mesolithic HGs. We observed that none of the target groups had a stronger affinity to Anatolian farmers than WHG, suggesting that the mixtures we date below reflect pre-Neolithic contacts between the HGs (Table S5.2).

To infer the timing of the mixtures in the history of Mesolithic European HGs, we applied DATES to hunter-gatherers from Scandinavia, the Baltic regions, and central Europe. We inferred that the earliest admixture occurred in Scandinavian HGs from Norway and Sweden around 80113 generations before the samples lived (Figure SB). Accounting for the average sampling age of the specimens and the mean human generation time of 28 years (1), this translates to a timing of admixture of $~ 10,200$ BCE for Norway and Sweden Mesolithic individuals, though dates are more recent $(\sim 8,000 \mathrm{BCE})$ in the Motala HG's. In the Baltic region, we inferred admixture dates of $~ 8,700-6,000$ BCE in Latvia and Lithuania HGs, postdating the mixture in Scandinavia (Figure 3). In southeast Europe, the Iron Gates region of the Danube Basin shows widespread evidence of mixtures between hunter-gatherer groups and, in the case of some outliers, mixture of huntergatherers and Anatolian farmer-related ancestry as early as the Mesolithic period (26). Further, these groups showed strong affinity to the WHG-related ancestry in Anatolian populations, suggesting ancient interactions with Near Eastern populations (26). We applied $q p A d m$ to test the model of admixture in Iron Gates HG and found that the parsimonious model with WHG and EHG provides a good fit to the data. Further, when we tested the model with Anatolian-related ancestry using Anatolian $\mathrm{HG}(\mathrm{AHG})$ as an additional source population, $\mathrm{AHG}$ was not required as the AHG 
ancestry proportion was not significant (Table S5.1.1 and S5.1.2). Applying DATES to Iron Gates HG with WHG and EHG as reference populations, we inferred this group was genetically formed in 10,000-8400 BCE. Our samples of the Iron Gates HGs include a wide range of C14 dates between 8,800-5,700 BCE. We confirmed our dates were robust to the sampling age of the individuals as we obtained statistically consistent dates when all samples were combined as one group or when subsets of samples were grouped in bins of 500 years (Figure SA). The most recent dates of 7,500 BCE were inferred in eastern Europe in Ukraine HGs, highlighting how the WHGEHG cline was formed over a period 2000-3000 years (Figure 3, Table SC).

Early to middle Neolithic: Neolithic farming began in the Near East - the Levant, Anatolia, and Iran - and spread to Europe and other parts of the world $(18,20,27)$. The first farmers of Europe were related to Anatolian farmers, whose origin remains unclear. The early Neolithic Anatolian farmers (Aceramic Anatolian farmers) had majority ancestry from AHG with some gene flow from the first farmers from Iran (26). AHG, in turn, had ancestry from Levant HG (Natufians) and some mysterious hunter-gatherer group related to the ancestors of WHG individuals from central Europe - a gene flow event that likely occurred in the late Pleistocene (26). Using qpAdm, we confirmed that early Anatolian farmers could be modeled as a mixture of AHG and Iran Neolithic farmer-related groups (Note S5). To learn about the timing of the genetic formation of early Anatolian farmers, we applied DATES using one reference group as a set of pooled individuals of WHG-related and Levant Neolithic farmers-related individuals as a proxy of AHG ancestry and the second reference group containing pooled Iran Neolithic farmer-related individuals. We note that the application of DATES to three-way admixed groups can lead to intermediate dates between the first and second pulse of gene flow unless the reference populations are chosen carefully (Table S2.1). Our setup for early Anatolian farmers should have limited confounding and should recover the timing of the most recent event (in this case, the gene flow from CHG or Iran Neolithic-related groups) reliably (Table S2.1). We infer the Iran Neolithic farmer-related gene flow occurred 10,900 BCE (12,200-9,600 BCE), predating the origin of farming in Anatolia (28). During the subsequent millennia, these early farmers further admixed with Levant Neolithic groups to form Anatolian Neolithic farmers who spread towards the west to Europe and in the east to mix with Iran Neolithic farmers, forming the Chalcolithic groups of Seh Gabi and Hajji Firuz. Using $D A T E S$, we inferred the Chalcolithic groups were genetically formed in $~ 7,600-5,700$ BCE (Table $\mathrm{SC})$.

In Europe, the Anatolian Neolithic farmers mixed with the local indigenous huntergatherers replacing between $\sim 3-50 \%$ ancestry of Neolithic Europeans. To elucidate the fine-scale patterns and regional dynamics of these mixtures, we applied DATES to time transect samples from 94 groups $(n=657)$ sampled from sixteen regions in Europe, ranging from $~ 6,000-1,900$ BCE and encompassing individuals from the early Neolithic to Chalcolithic periods (Table SB). Using qpAdm, we first confirmed that the Neolithic Europeans could be modeled as a mixture of European hunter-gatherer-related ancestry and Anatolian farmer-related ancestry and inferred their ancestry proportions (Table SD). For most target populations ( 80\%), we found the model of gene 
flow between Anatolian farmer-related and WHG-related ancestry provided a good fit to the data ( $p$-value $>0.05$ ). In some populations, we found variation in the source of the HG-related ancestry and including either EHG or GoyetQ2 improved the fit of the model. In five groups, none of the models fit, despite excluding outlier individuals whose ancestry profile differed from the majority of the individuals in the group (Table SD, Table SE). To confirm that the target populations do not harbor Steppe pastoralist-related ancestry, we applied $D$-statistics of the form $D$ (Mbuti, target, Anatolian farmers, Steppe pastoralists) where target $=$ Neolithic European groups. We observed that four groups had a stronger affinity to Steppe pastoralists compared to Anatolian farmers, and hence we excluded these from further analysis (Table SF). After filtering, we applied DATES to 86 European Neolithic groups using WHG-related individuals and Anatolian farmers as reference populations.

Earlier analysis has suggested that farming spread along two main routes in Europe, from southeast to central Europe ('continental route') and along the Mediterranean coastline to Iberia ('coastal route') $(23,29,30)$. Consistent with this, we inferred one of the earliest timings of gene flow in the Balkans around 6,400 BCE. Using the most comprehensive time-transect in Hungary with 19 groups $(n=63)$ spanning from middle Neolithic to late Chalcolithic, we inferred that the admixture occurred between $\sim 6,100-4,500$ BCE. Under a model of a single shared gene flow event in the common ancestors of all individuals, we would expect to obtain similar dates of admixture (before present) after accounting for the age of the ancient specimens. Similar to Lipson et al. (2017), we observed that the estimated dates in middle Neolithic individuals were substantially older than those inferred in late Neolithic or Chalcolithic individuals (Figure 3). This would be expected if the underlying model of gene flow involved multiple pulses of gene flow, such that the timing in the middle Neolithic samples reflects the initial two-way mixture and the timing in the Chalcolithic samples captures both recent and older events. Interestingly, Lipson et al. (2017) and other recent studies have documented increasing HG ancestry from $\sim 3-15 \%$ from the Neolithic to Chalcolithic period $(16,23,31)$, suggesting that there was additional $\mathrm{HG}$ gene flow after the initial mixture. This highlights that the interactions between local hunter-gatherers and incoming Anatolian farmers were complex with multiple gene flow events between these two groups, which explains the increasing HG ancestry and more recent dates in Chalcolithic individuals (Table SD).

Mirroring the pattern in Hungary, we documented the resurgence of HG ancestry in the Czech Republic, France, Germany, and southern Europe. In central Europe, we inferred that the Anatolian farmer-related gene flow occurred $~ 5,600-5,000$ BCE, with some exceptions. In the Blätterhöhle site from Germany, we inferred the gene flow occurred more recently ( 4,000 BCE), consistent with the occupation of both hunter-gatherers and farmers in this region until the late Neolithic (31). In eastern Europe, using samples related to the Funnel Beaker culture (TRB; from German Trichterbecher) from Poland, we dated the Anatolian farmer-related gene flow occurred 5,300-4,200 BCE. Following the TRB decline, the Baden culture and the Globular Amphora culture appeared in many areas of Poland and Ukraine (25). These cultures had close contacts with

Corded Ware complex and steppe societies, though we did not find any evidence of Steppe pastoralist-related ancestry in the GAC individuals (Table SD). Applying DATES, we inferred the 
Anatolian farmer-related and HG mixture occurred 5,200-3,100 BCE, predating the spread of Steppe pastoralists to eastern Europe $(16,19)$.

Along the Mediterranean route, we characterized Anatolian farmer-related gene flow in Italy, Iberia, France, and the British Isles. Using samples from five groups in Italy, we inferred the earliest dates of Anatolian farmer-related gene flow of $\sim 6,100 \mathrm{BCE}$, and within the millennium, the ancestry spread from Sardinia to Sicily (Figure 3). In Iberia, the Anatolian farmer-related mixture occurred $~ 6,000-3,400 \mathrm{BCE}$ and showed evidence for an increase in HG ancestry from 9-20\% after the initial gene flow. In France, previous studies have shown that Anatolian farmerrelated ancestry came from both routes, along the Danubian in the north and along the Mediterranean in the south (23). This is reflected in the source of the HG ancestry, which is predominantly EHG and WHG-related in the north and includes WHG and Goyet-Q2 ancestry in the south (23). Consistently, we also observed that the admixture dates in France were structured along these routes, with the median estimate of $\sim 5,100 \mathrm{BCE}$ in the east and much older $\sim 5,500$ BCE in the south (Table SC). In Scandinavia, we inferred markedly more recent dates of admixture of $\sim 4,300$ BCE using samples from Sweden associated with the TRB culture and Ansarve Megalithic tombs, consistent with a late introduction of farming to Scandinavia (33).

Finally, we inferred recent dates of admixture in Neolithic samples from the British Isles

Late Neolithic to Bronze Age: The beginning of the Bronze Age was a period of major cultural and demographic change in Eurasia, accompanied by the spread of Yamnaya Steppe Pastoralistrelated ancestry from Pontic-Caspian steppes into Europe and South Asia (16). The archaeological record documents that the early Steppe pastoralists cultures of Yamnaya and Afanasievo, with characteristic burial styles and pottery, appeared around $\sim 3,300$ to 2,600 BCE (35). These groups were likely the result of a genetic admixture between the descendants of EHG-related groups and CHG-related groups associated with the first farmers from Iran $(8,22,36)$. Using $q p A d m$, we first tested how well this model fits the data from 8 early Steppe pastoralist groups, including seven groups associated with Yamnaya culture and one group related to the Afanasievo culture (Methods). For all but two Yamnaya groups (from Hungary Baden and Russia Kalmykia), we found this model provides a good fit to the data (Table S5.4). We note that the samples from of the formation of the early Steppe pastoralist-related groups, we applied DATES using pooled EHG and pooled Iranian Neolithic farmers. Focusing on the groups with the largest sample sizes, Yamnaya Samara $(n=10)$ and Afanasievo $(n=19)$, we inferred the admixture occurred between 40 
45 generations before the individuals lived, translating to an admixture timing of $\sim 4,100 \mathrm{BCE}$ (Table S6.1). We obtained qualitatively similar dates across four Yamnaya and one Afanasievo groups, consistent with the findings that these groups descend from a recent common ancestor (for Ozera samples from Ukraine, the dates were not significant). This is also further supported by the insight that the genetic differentiation across early Steppe pastoralist groups is very low ( $F_{S T} \sim$ 0.000-0.006) (Table S6.2). Thus, we combined all early Steppe pastoralist individuals in one group to obtain a more precise estimate for the genetic formation of proto-Yamnaya of $\sim 4,400$ to 4,000 BCE (Figure 2). These dates are noteworthy as they pre-date the archaeological evidence by more than a millennium (37) and have important implications for understanding the origin of protoPontic Caspian cultures and their spread to Europe and South Asia. (CWC) and Bell Beaker complex (BBC) cultures brought Steppe pastoralist-related ancestry to Europe. Present-day Europeans derive between $~ 10-60 \%$ Steppe pastoralist-related ancestry, which was not seen in Neolithic samples. To obtain a precise chronology of the spread of Steppe pastoralist-related ancestry across Europe, we analyzed 109 late Neolithic, Chalcolithic, and BA samples dated between 3,000-750 CE from 18 regions, including samples associated with the CWC and BBC cultures. We first confirmed that most target samples had Steppe pastoralist-related ancestry, in addition to European HG-related and Anatolian farmer-related ancestry using qpAdm. We excluded 20 groups that could not be parsimoniously modeled as a three-way mixture even after removing individual outliers. After filtering, we retained 79 groups for dating Steppe pastoralist-related gene flow across Europe (Note S5, Table SH). As Bronze Age Europeans have ancestry from three distinct groups, we applied DATES using the following two reference populations, one group including early Steppe pastoralists (Yamnaya and Afanasievo) and the other group with pooled samples of WHG-related and Anatolian farmer-related individuals, which is the proxy for the ancestral Neolithic Europe population.

To learn about the spread of CWC culture across Europe, we used seven late Neolithic and Bronze age groups, including five associated with CWC artifacts. Using DATES, we inferred that the oldest date of Steppe pastoralists gene flow in Europe was 3,200 BCE in Scandinavia in samples associated with Battle Axe Culture in Sweden and Single Grave Culture in Denmark that were both contemporary to CWC. The samples from Scandinavia showed large heterogeneity in ancestry, including some individuals with majority Steppe pastoralist-related ancestry (and negligible amounts of Anatolian farmer-related ancestry), consistent with patterns expected from recent gene flow (38). Strikingly, we inferred the timing of admixture in central Europe (Germany and the Czech Republic) and eastern Europe (Estonia and Poland) to be remarkably similar. These dates fall within a narrow range of $\sim 3,000-2,900 \mathrm{BCE}$ across diverse regions, suggesting that the mixed population associated with the Corded Ware culture formed over a short time and spread across Europe rapidly with very little further mixture (Table SC).

Following the Corded Ware culture, from around 2,800 to 2,300 BCE, Bell Beaker pottery became widespread across Europe (39). Using 19 Chalcolithic and Bronze Age samples, including ten associated with Beaker-complex artifacts, we inferred the dynamics of the spread of the Beaker 
complex across Europe. We inferred the oldest date of Steppe pastoralist-related admixture was 3,200 BCE (3600-2800 BCE) in EBA Mallorca samples from Iberia. We note the EBA Mallorca sample is not directly associated with Beaker culture, but qpAdm modeling suggests that this individual is clade with the small subset of Iberian Beaker-complex-associated individuals who carried Steppe pastoralist-related (40). Most individuals from Iberia, however, had negligible Steppe pastoralist-related ancestry suggesting the Beaker culture was not accompanied by major gene flow in Iberia despite the earliest dates (Table SH). In central and western Europe, where steppe gene flow was more pervasive, we inferred the median date of the mixture was $\sim 2,700 \mathrm{BCE}$ with the oldest dates in the Netherlands, followed by Germany and France (Figure 3). There was, however, large heterogeneity in the dates across Europe and even within the same region. For example, comparing two BA groups from the Netherlands suggests a wide range of dates $\sim 3,000$ $\mathrm{BCE}$ and 2,500 BCE, and four groups from Germany indicate a range of 2,900-2,700 BCE. From central Europe, the Steppe pastoralist-related ancestry spread quickly to the British Isles, where people with steppe ancestry replaced $90 \%$ of the genetic ancestry of individuals from Britain. Our estimates for the time of gene flow in Bell Beakers samples from England suggest that the gene flow occurred 2,700 BCE (2770-2550 BCE). Our estimated dates of admixture are older than the dates of arrival of this ancestry in Britain (41) and, interestingly, overlap the dates in central Europe. Given that a significant fraction of the Beaker individuals were recent migrants from central Europe, we interpret our dates reflect the admixture into ancestors of the British Beaker people, occurring in mainland Europe (41).

The middle to late Bronze age led to the final integration of Steppe pastoralist-related ancestry in Europe. In southern Europe, early BA samples had limited Steppe pastoralist-related ancestry, though present-day individuals have between $\sim 5-30 \%$ steppe ancestry (16). Using pooled samples of middle to late BA from Spain, we inferred major mixture occurred 2,500 BCE in Iberia. We inferred a similar timing in Italy using individuals associated with the Bell Beaker culture and early BA samples from Sicily (Table SC). In Sardinia, a majority of the BA samples do not have Steppe pastoralist-related ancestry. In a few individuals, we found evidence for steppe ancestry, though in most cases, the Steppe pastoralist-related ancestry proportion overlapped 0 , and the dates were very noisy (Table SH). Using Iron Age samples from Sardinia, we inferred the gene flow occurred $\sim 2,600 \mathrm{BCE}$, though there is large uncertainty associated with this estimate $(2,614+/-560 \mathrm{BCE})$. In other parts of continental Europe and the British Isles, the Steppe pastoralist-related gene flow got diluted over time, as evidenced by more recent dates in LBA than EBA or MBA samples in Germany, England, and Scotland, and increase in Neolithic farmer ancestry during this period (42) (Table SC).

Finally, the Corded Ware Complex expanded to the east to form the archaeological complexes of Sintashta, Srubnaya, Andronovo, and the Bronze Age cultures of Kazakhstan. Samples associated with these cultures harbor mixed ancestry from the Yamnaya Steppe pastoralist-related groups (CWC, in some cases) and Neolithic individuals from central Europe groups, we inferred the precise timing for the formation of these groups beginning in the third 
millennium BCE. These groups were formed chronologically, with the date of genetic formation of 3,200 BCE for Sintashta culture, followed by 2,900 BCE for Srubnaya and Andronovo cultures. In the central Steppe region (present-day Kazakhstan), we obtained median dates of $\sim 2,800$ BCE for the expansion of Steppe pastoralist-related ancestry in four Kazakh cultures of Maitan Alakul, Aktogai, and Kairan. By 2,700 BCE, most of these cultures had almost 60-70\% Yamnaya Steppe pastoralist-related ancestry (Table SC). These groups, in turn, expanded eastwards, transforming the genetic composition of populations in South Asia.

\section{Discussion}

We developed DATES, a novel method to measure ancestry covariance in a single diploid individual genome to estimate the time of admixture. Using extensive simulations, we show that DATES provides accurate estimates of the timing of admixture for a range of demographic scenarios. Application of DATES to present-day samples shows that the results are concordant with published methods - Rolloff, ALDER, and Globetrotter. For sparse datasets, DATES outperforms published methods as it does not require phased data and works reliably with limited samples, large proportions of missing variants as well as pseudodiploid genotypes. This makes DATES ideally suited for the analysis of ancient DNA samples.

We illustrate the application of DATES by reconstructing population movements and admixtures during the European Holocene. The European continent was subject to two major migrations during the Holocene: the movement of Anatolian farmers during the Neolithic and the migration of Yamnaya Steppe pastoralists during the Bronze Age. First, we document that the Mesolithic hunter-gatherers formed as a mixture of WHG and EHG ancestry 10,200 to 7400 BCE. These dates are consistent with the archeological evidence for the appearance of lithic technology associated with eastern HGs in Scandinavia and the Baltic regions and the spread of WHG ancestry to east $(17,43,44)$. Next, we studied the timing of the genetic formation of Anatolian farmers. The earliest evidence of agriculture comes from the Fertile Crescent, the southern Levant, and the Zagros Mountains of Iran and dates to around 10,000 BCE. In central Anatolia, farming has been documented c. 8,300 BCE $(45,46)$. It has been long debated if Neolithic farming groups from Iran and the Levant introduced agriculture to Anatolia or huntergatherers in the region locally adopted agricultural practices. The early Anatolian farmers can be modeled as a mixture of local hunter-gatherers people related to Caucasus hunter-gatherers or first farmers from Iran (26). By applying DATES (assuming single instantaneous admixture), we inferred that the Iran Neolithic gene flow occurred around 10,900 BCE ( 12,200-9,600 BCE). An alternate possibility is that there was a long period of gradual gene flow between the two groups and our dates reflect intermediate dates between the start and end of the gene flow. An upper bound for such mixture comes from the lack of Iran Neolithic ancestry in Anatolian HGs at 13,000 BCE, and a lower bound comes from the $\mathrm{C} 14$ dates of early Anatolian farmers, one of which is directly dated at 8269-8210 BCE (26). In either case (instantaneous admixture or gradual gene flow), the genetic mixture that formed Anatolian farmers predates the advent of agriculture in this region. This supports the model that Anatolian hunter-gatherers locally transitioned to agricultural 
subsistence, and most probably, there was cultural diffusion from other regions in Near East (Iran and Levant) (26). Future studies with more dense temporal sampling will shed light on the demographic processes that led to the transition from foraging to farming in the Near East, and in turn, elucidate the relative roles of demic and cultural diffusion in the dispersal of technologies like agriculture across populations.

Using data from sixteen regions in Europe, we reconstruct a detailed chronology and dynamics of the expansion and admixture of Anatolian farmers during the Neolithic period. We infer that starting in $~ 6,400 \mathrm{BCE}$, gene flow from Anatolian farmers became widespread across Europe, and by $\sim 4,300 \mathrm{BCE}$, it was present in almost all parts of continental Europe and the British Isles. These dates are significantly more recent than the estimates of farming based on archaeological evidence in some parts of Europe, suggesting that the local hunter-gatherers and farmers co-existed for more than a millennium before the mixture occurred $(16,31)$. In many regions, after the initial mixture, there was a resurgence of $\mathrm{HG}$ ancestry, highlighting the complexities of these ancient interactions. We note that our results are consistent with two previous genetic studies, Lipson et al. (2017) and Rivollat et al. (2020), that applied genetic dating methods to a subset of samples we used in our analysis. Lipson et al. (2017) used a modified version of ALDER to infer the timing of admixture in three regions $(n=151)$, and we obtained statistically consistent results for all overlapping samples (within two standard errors). An advantage of our approach over the modified ALDER approach is that we do not rely on helper samples (higher coverage individuals combined with the target group) for dating; unless these have a similar ancestry profile, they could bias the inferred dates. Our results are concordant with Rivollat et al. (2020) that used a previous version of DATES to infer the timing of Neolithic gene flow in 32 groups (vs. 86 groups in our study). We find the performance of both versions of DATES is similar, though some implementation details have improved (see Note S3, Table S3.3).

The second major migration occurred when populations associated with the Yamnaya culture in the Pontic-Caspian steppe expanded to central and western Europe from far eastern Europe. Our analysis reveals the precise timing of the genetic formation of these early Steppe pastoralists groups-Yamnaya and Afanasievo-occurred $~ 4,400-4,000$ BCE. This estimate predates the archaeological evidence by more than a millennium (37) and suggests the presence of an ancient "ghost" population of proto-Yamnaya around this time. Understanding the source and location of this ghost population will provide deep insights into the history of Pontic-Caspian cultures and the origin of Indo-European languages that have been associated to have spread with Steppe pastoralists ancestry to Europe and South Asia (16, 47). Starting in $~ 3,200$ BCE, the Yamnaya-derived cultures of Corded Ware Complex and Bell Beaker complex spread westwards, bringing steppe ancestry to Europe. Our analysis reveals striking differences in the spread of these three cultures: the Yamnaya were genetically formed a millennium before the evidence for pastoralism, while CWC formation is coincident with the archaeological dates and similar across diverse regions separated by thousands of kilometers, suggesting a rapid spread after the initial formation of this group. In contrast, the formation and expansion of people with Steppe pastoralistrelated ancestry associated with Bell Beakers cultural artifacts are much more complex and 
heterogeneous across regions. We find the earliest evidence of Steppe pastoralist-related ancestry in Iberia around $3200 \mathrm{BCE}$, though this ancestry only becomes widespread after 2,500 BCE. In central Europe, the gene flow occurred simultaneously with archaeological evidence and was coexisting with the Corded Ware complex in some parts $(41,48)$. Finally, in the British Isles, the Bell Beaker culture spreads rapidly from central Europe and replaces almost $90 \%$ of the ancestry of individuals in this region (41).

Recent analysis has shown remarkable parallels in the history of Europe and South Asia; with both groups deriving ancestry from local indigenous HGs, Near Eastern farmers, and Steppe pastoralist-related groups (8). Interestingly, however, the timing of the two major migrations events differs across the two subcontinents. Both mixtures occurred in Europe almost a millennium before they occurred in South Asia. In Europe, the Neolithic migrations primarily involved Anatolian farmers, while the source of Neolithic ancestry is closer to Iran Neolithic farmers in South Asia. The Steppe pastoralist-related gene flow occurred in the context of the spread of CWC and BBC cultures in Europe around 3,200-2,500 BCE; in South Asia, this ancestry arrived with Steppe MLBA cultures in 1,800-1,500 BCE (8). The Steppe MLBA groups were genetically formed as an admixture of Steppe pastoralist-derived groups and European Neolithic farmers following the eastward expansion of CWC groups between 3,200-2,700 BCE. Understanding the origin and migration paths of the ancestral groups thus helps to illuminate the differences in the timeline of the spread of steppe genetics across the two subcontinents of Eurasia.

Genomic dating methods like DATES provide an independent and complementary approach for reconstructing population history. By focusing on genetic clocks like recombination rate, we provide an independent estimate of the timing of evolutionary events up to several thousands of years. Our analysis also has advantages over temporal sampling of ancient DNA, in that we can obtain direct estimates of when a population was formed, rather than inferring putative bounds for the timing based on the absence/presence of a particular ancestry signature (which may be sensitive to sampling choice and density). Genetic approaches provide complementary evidence to archaeology and linguistics as they date the time of gene flow and not migration. Both dates are similar in many contemporary populations like African Americans and Latinos, though this may not be generally true (2). This is underscored by our dates for the Neolithic farmer mixture, which post-dates evidence of material culture related to agriculture by almost two millennia in some regions. This suggests that European HGs and farmers resided side by side for several thousand years before gene exchange $(49,50)$. This highlights how genetic dates can provide complementary evidence to archaeology and help to build a comprehensive picture of population origins and movements.

\section{Methods and Materials}

605

\section{Dataset}

607

We analyzed 1,096 ancient European samples from 152 groups restricting to data from 1,233,013 autosomal SNP positions that were genotyped using the Affymetrix Human Origins array (the 
V44.3 release of the Allen Ancient DNA Resource (AADR); https://reich.hms.harvard.edu/allenfiltered this dataset to remove samples that were marked as contaminated, low coverage, outliers, duplicates or first- or second-degree relatives (Table SB). We grouped individuals together from

612 a particular culture or region. Details of sample affiliation and grouping used is described in Table

613 SA.

\section{Modeling admixture history}

616

We applied $q p A d m$ from ADMIXTOOLS to identify the best fitting model and estimate the ancestry proportions in a target population modeled as a mixture of $n$ "reference" populations using a set of "Outgroup" populations (16). We set the details: YES parameter, which reports a normally distributed Z-score to evaluate the goodness of fit of the model (estimated with a Block Jackknife). For each target population, we chose the most parsimonious model, i.e., fitting the data with the minimum number of source populations. We excluded models where the $p$-value $<0.05$ indicating a poor fit to the data. Details of the $q p A d m$ analysis for each group are reported in Note S5. We also applied $D$-statistics in some cases using qpDstat in ADMIXTOOLS with default parameters.

\section{Dating admixture events}

627

We applied DATES to infer the time of admixture for a given target population. We present the details of the model and implementation in Note S1. We applied DATES using genome-wide SNP data from the target population and two reference populations. To infer the allele frequency in the ancestral populations more reliably, where specified, we pooled individuals deriving the majority of their ancestry from the population of interest (Table SA). We computed the weighted ancestry covariance between $0.45 \mathrm{cM}$ (to minimize the impact of background LD) to $100 \mathrm{cM}$, with a bin size of $0.1 \mathrm{cM}$. We plotted the weighted covariance with genetic distance and obtained a date by fitting an exponential function with an affine term $y=A e^{-\lambda d}+c$, where $d$ is the genetic distance in Morgans and $\lambda=(t+1)$ is the number of generations since admixture $(t)$. The factor of $(t+1)$ is because in the first-generation following admixture, the admixed population derives one chromosome from each ancestral group. The mixing of chromosomes only begins in the following generations as the chromosomes recombine. We computed standard errors using weighted block jackknife, where one chromosome was removed in each run (51). We examined the quality of the exponential fit by computing the normalized root-mean-square deviation (NRMSD) between the 643 (11).

644 The estimated dates of admixture were considered significant if the $Z$-score $>2, \lambda<200$ 645 generations and NRMSD < 0.7. We converted the inferred dates from generations to years by 646 assuming a mean generation time of 28 years (1). For ancient samples, we added the sampling age 647 of the ancient specimen (Table SA). When multiple individuals were available, we used the 
648 average sampling ages to offset the admixture dates. We report dates in BCE by assuming the 1950

649 convention.

650

\section{Software availability}

652

653 The executable and source code for DATES will be available on GitHub:

654 https://github.com/MoorjaniLab/DATES_v3600

655

\section{Acknowledgments}

657

We thank Monty Slatkin, Ziyue Gao, David Reich, Iosif Lazaridis, and Vagheesh Narasimhan for

659 their comments on the manuscript. We thank Iosif Lazaridis for helpful discussions about

660 population models in the Near East, Remi Tournebize for suggestions for evaluating the fit of 661 exponential decay curves, and Neel Alex for suggestions for implementation of FFT for an earlier 662 version of DATES. We are grateful for support from the Burroughs Wellcome Fund Careers at the 663 Scientific Interface, Sloan Research Fellowship, and NIH R35GM142978 awarded to PM. NP was 664 a fellow at the Radcliffe Institute for Advanced Study at Harvard University. 


\section{Figures}

666

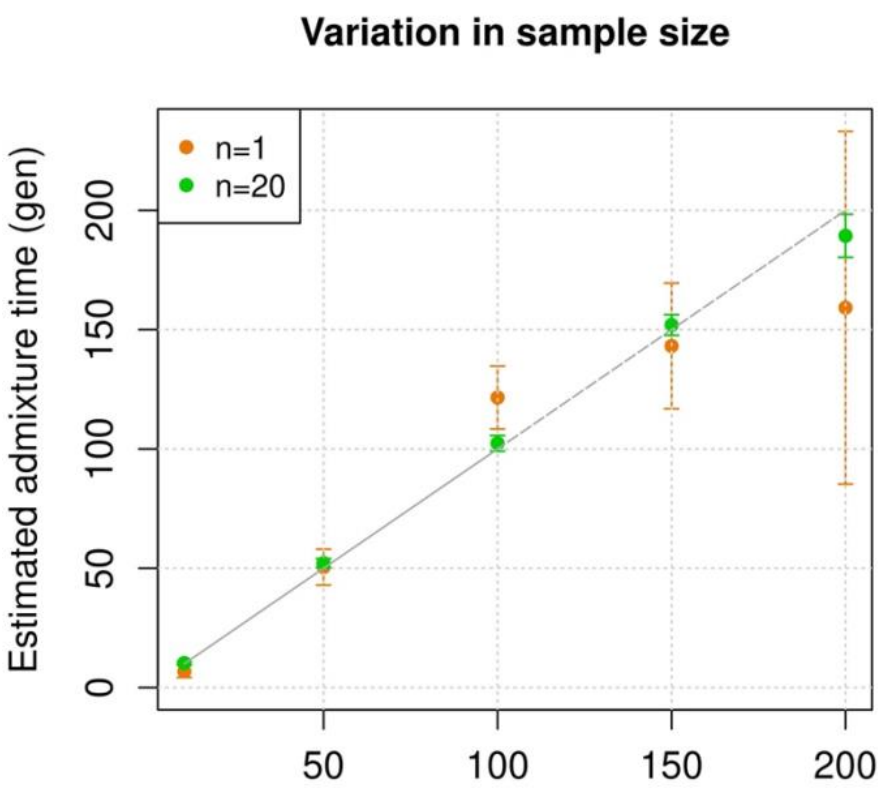

True admixture time (gen)

668 Figure 1: Simulation results. We constructed admixed individuals with $20 \%$ European (CEU) 669 and $80 \%$ Africa (YRI) ancestry for admixture dates ranging between 10-200 generations where 670 the sample size of the target group is as shown in the legend. We applied DATES using French and 671 Yoruba as reference populations. We show the true time of admixture (X-axis, in generations) and 672 the estimated time of admixture ( \pm 1 SE) (Y-axis, in generations). Standard errors were calculated 673 using a weighted block jackknife approach by removing one chromosome in each run (Methods). 

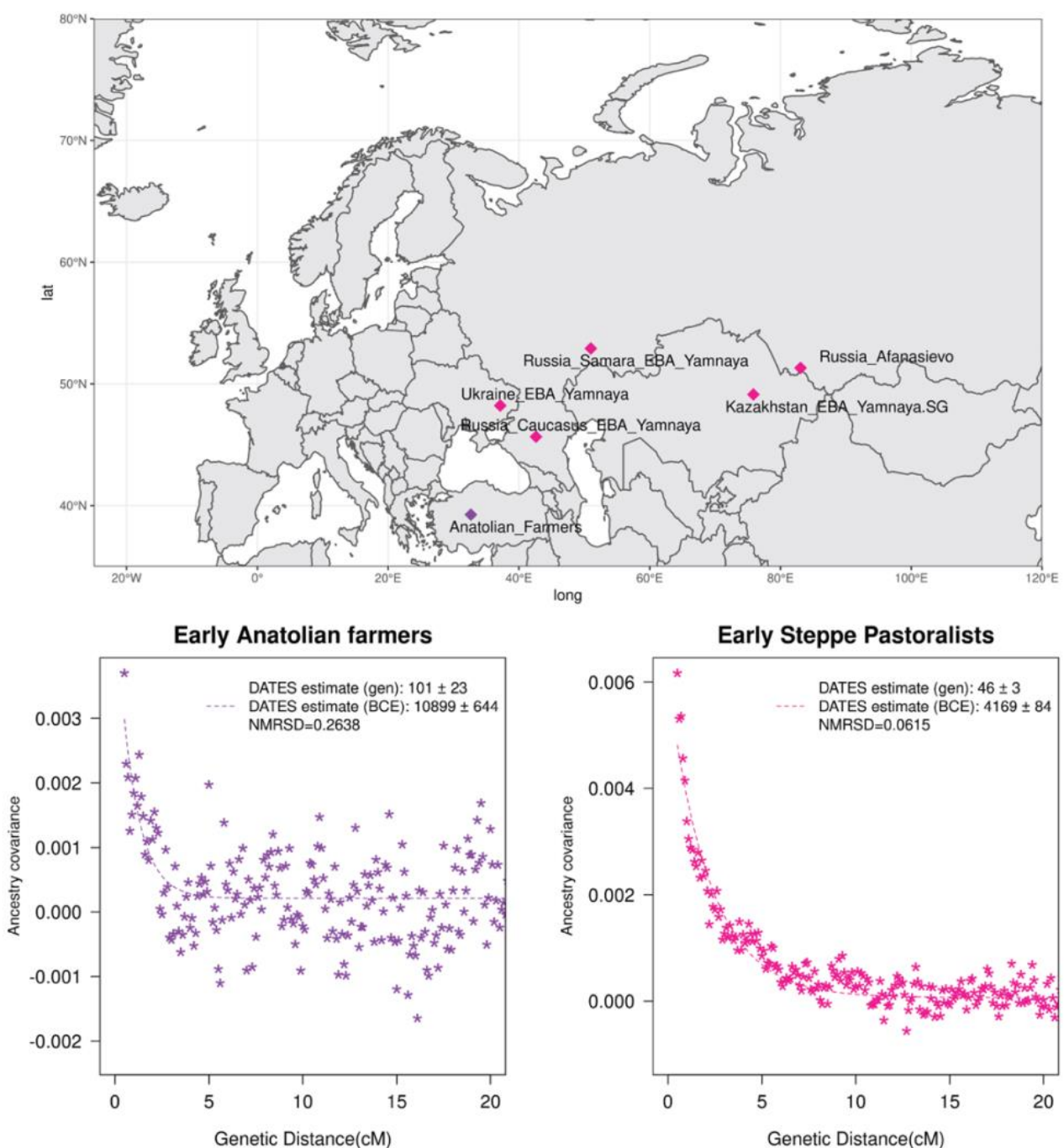

674

675

676

677

678

679

680

681

682

683

684

685

686

687

688

689

690

691

692
Figure 2: Genetic formation of early Anatolian farmers and early Bronze Age Steppe pastoralists. The top panel shows a map with sampling locations of the target groups analyzed for admixture dating. The bottom panels show the inferred times of admixture for each target using $D A T E S$ by fitting an exponential function with an affine term $y=A e^{-\lambda d}+c$, where $d$ is the genetic distance in Morgans and $\lambda=(t+1)$ is the number of generations since admixture $(t)$ (Methods). We start the fit at a genetic distance $(d)>0.5 \mathrm{cM}$ to minimize confounding with background LD and estimate a standard error by performing a weighted block jackknife removing one chromosome in each run. For each target, in the legend, we show the inferred average dates of admixture $( \pm 1 \mathrm{SE})$ in generations before the individual lived, in BCE accounting for the average age of all the individuals and the mean human generation time, and the NRMSD values to assess the fit of the exponential curve (Methods). The bottom left shows the ancestry covariance decay curve for early Anatolian farmers inferred using one reference group as a set of pooled individuals of WHG-related and Levant Neolithic farmers-related individuals as a proxy of AHG ancestry and the second reference group containing Iran Neolithic farmer-related individuals. The bottom right shows the ancestry covariance decay curve for early Steppe pastoralists groups, including all Yamnaya and Afanasievo individuals as the target group and EHG-related and Iran Neolithic farmer-related groups as reference populations. 

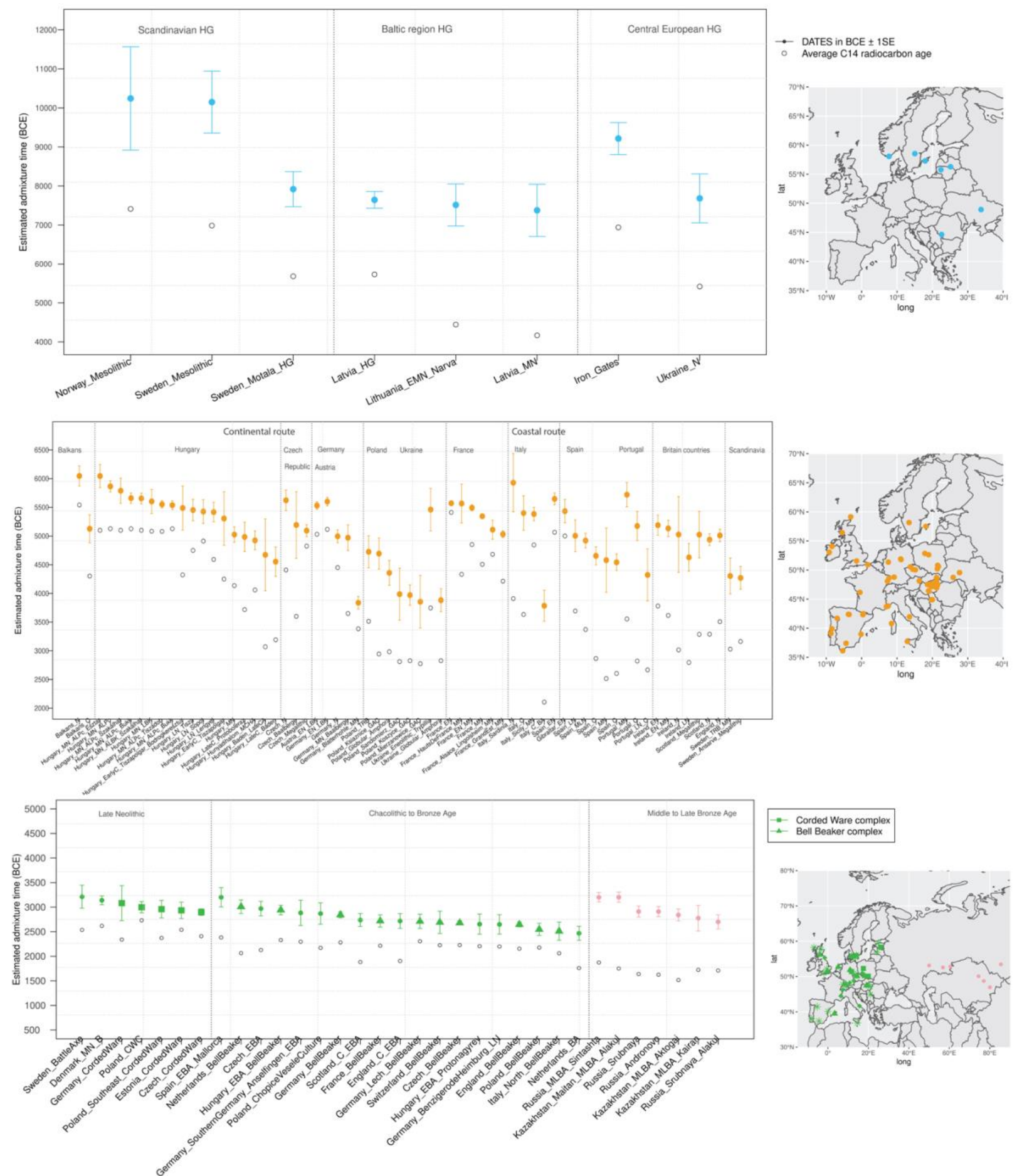

694 Figure 3: Timeline of admixture events in ancient Europe. We applied DATES to ancient 695 samples from Europe. In the right panel, we show the sampling locations of the ancient specimens, and in the left panel, we show the admixture dates for each target group listed on the X-axis. The inferred dates in generations were converted to dates in BCE by assuming a mean generation time of 28 years (4) and accounting for the average sampling age (shown as grey dots) of all ancient individuals in the target group (Methods). The top panel shows the formation of WHG-EHG cline (in blue) using Mesolithic hunter-gatherers as the target and EHG and WHG as reference 
populations. The middle panel shows admixture dates of local HGs and Anatolian farmers (in orange) using Neolithic European groups as targets and Anatolian farmers-related groups and WHG-related groups as reference populations. The bottom panel shows the spread of Steppe pastoralist-related ancestry (in green) estimated using middle and late Neolithic, Chalcolithic, and Bronze Age samples from Europe as target populations and early Steppe pastoralist-related groups (Afanasievo and Yamnaya Samara) and a set of Anatolian farmers and WHG-related groups as reference populations. For the middle to late Bronze Age samples from Eurasia, we used the early Steppe pastoralist-related groups and the Neolithic European groups as reference populations. The cultural affiliation (CWC, BBC, or Steppe MLBA cultures) of the individuals is shown in the legend. 


\section{References}

1. P. Moorjani, et al., A genetic method for dating ancient genomes provides a direct estimate of human generation interval in the last 45,000 years. Proc. Natl. Acad. Sci. 113, 5652 (2016).

2. G. Hellenthal, et al., A Genetic Atlas of Human Admixture History. Science 343, 747-751 (2014).

3. R. Chakraborty, K. M. Weiss, Admixture as a tool for finding linked genes and detecting that difference from allelic association between loci. Proc. Natl. Acad. Sci. 85, 9119-9123 (1988).

4. P. Moorjani, et al., The History of African Gene Flow into Southern Europeans, Levantines, and Jews. PLoS Genet. 7, e1001373 (2011).

5. P.-R. Loh, et al., Inferring Admixture Histories of Human Populations Using Linkage Disequilibrium. Genetics 193, 1233-1254 (2013).

6. S. Gravel, Population Genetics Models of Local Ancestry. Genetics 191, 607-619 (2012).

7. A. Auton, et al., A global reference for human genetic variation. Nature 526, 68-74 (2015).

8. V. M. Narasimhan, et al., The formation of human populations in South and Central Asia. Science 365, eaat7487 (2019).

9. N. A. Rosenberg, et al., Genetic Structure of Human Populations. Science 298, 2381 (2002).

10. N. Patterson, et al., Ancient Admixture in Human History. Genetics 192, 1065-1093 (2012).

11. R. Tournebize, G. Chu, P. Moorjani, Reconstructing the history of founder events using genome-wide patterns of allele sharing across individuals. bioRxiv, 2020.09.07.286450 (2020).

12. D. M. Behar, et al., The genome-wide structure of the Jewish people. Nature 466, 238-242 (2010).

13. B. M. Henn, et al., Genomic Ancestry of North Africans Supports Back-to-Africa Migrations. PLoS Genet. 8, e1002397 (2012).

14. L. Pagani, V. Colonna, C. Tyler-Smith, Q. Ayub, An Ethnolinguistic and Genetic Perspective on the Origins of the Dravidian-Speaking Brahui in Pakistan. 13 (2017).

15. A. L. Price, et al., Sensitive Detection of Chromosomal Segments of Distinct Ancestry in Admixed Populations. PLoS Genet. 5, e1000519 (2009). 
16. W. Haak, et al., Massive migration from the steppe was a source for Indo-European languages in Europe. Nature 522, 207-211 (2015).

17. I. Mathieson, et al., The genomic history of southeastern Europe. Nature 555, 197-203 (2018).

18. P. Skoglund, et al., Origins and Genetic Legacy of Neolithic Farmers and Hunter-Gatherers in Europe. Science 336, 466-469 (2012).

19. M. E. Allentoft, et al., Population genomics of Bronze Age Eurasia. Nature 522, 167-172 (2015).

20. W. Haak, et al., Ancient DNA from European Early Neolithic Farmers Reveals Their Near

21. Z. Hofmanová, et al., Early farmers from across Europe directly descended from Neolithic Aegeans. Proc. Natl. Acad. Sci. 113, 6886-6891 (2016).

22. E. R. Jones, et al., Upper Palaeolithic genomes reveal deep roots of modern Eurasians. Nat. Commun. 6, 8912 (2015).

23. M. Rivollat, et al., Ancient genome-wide DNA from France highlights the complexity of interactions between Mesolithic hunter-gatherers and Neolithic farmers. Sci. Adv. 6, eaaz5344 (2020).

24. Q. Fu, et al., The genetic history of Ice Age Europe. Nature 534, 200-205 (2016).

25. D. M. Fernandes, et al., A genomic Neolithic time transect of hunter-farmer admixture in central Poland. Sci. Rep. 8, 14879 (2018).

26. M. Feldman, et al., Late Pleistocene human genome suggests a local origin for the first

28. B. Bramanti, et al., Genetic discontinuity between local hunter-gatherers and central

29. D. Gronenborn, The persistence of hunting and gathering: Neolithic western temperate and Central Europe, in The Oxford Handbook of the Archaeology and Anthropology of HunterGatherers, V. Cummings, P. Jordan, M. Zvelebil, Eds. (Oxford University Press, 2014).

30. J. Guilaine, Aspects de la néolithisation en Méditerranée et en France, in The Widening Harvest. The Neolithic Transition in Europe: Looking Back, Looking Forward, A. 
31. M. Lipson, et al., Parallel palaeogenomic transects reveal complex genetic history of early European farmers. Nature 551, 368-372 (2017).

32. Marija Gimbutas, The Living Goddesses. (University of California Press., 2001).

33. A. Mittnik, et al., The genetic prehistory of the Baltic Sea region. Nat. Commun. 9, 442 (2018).

34. S. Brace, et al., Ancient genomes indicate population replacement in Early Neolithic Britain. Nat. Ecol. Evol. 3, 765-771 (2019).

35. N. L. Morgunova, O. S. Khokhlova, Chronology and Periodization of the Pit-Grave Culture in the Region Between the Volga and Ural Rivers Based on Radiocarbon Dating and Paleopedological Research. Radiocarbon 55, 1286-1296 (2013).

36. C.-C. Wang, et al., Ancient human genome-wide data from a 3000-year interval in the Caucasus corresponds with eco-geographic regions. Nat. Commun. 10, 590 (2019).

37. D. W. ANTHONY, The Horse, the Wheel, and Language (Princeton University Press, 2007) https:/doi.org/10.2307/j.ctt7sjpn (December 13, 2020).

38. H. Malmström, et al., The genomic ancestry of the Scandinavian Battle Axe Culture people and their relation to the broader Corded Ware horizon. Proc. R. Soc. B Biol. Sci. 286, 20191528 (2019).

39. Fokkens, H. and Nicolis, F, Background to Beakers. Inquiries into regional cultural backgrounds of the Bell Beaker complex. (Leiden: Sidestone Press., 2012).

40. D. M. Fernandes, et al., The spread of steppe and Iranian-related ancestry in the islands of the western Mediterranean. Nat. Ecol. Evol. 4, 334-345 (2020).

41. I. Olalde, et al., The Beaker phenomenon and the genomic transformation of northwest Europe. Nature 555, 190-196 (2018).

42. I. Olalde, et al., The genomic history of the Iberian Peninsula over the past 8000 years. Science 363, 1230-1234 (2019).

43. N. Kashuba, et al., Ancient DNA from mastics solidifies connection between material culture and genetics of mesolithic hunter-gatherers in Scandinavia. Commun. Biol. 2, 185 (2019).

44. T. Günther, et al., Population genomics of Mesolithic Scandinavia: Investigating early postglacial migration routes and high-latitude adaptation. PLOS Biol. 16, e2003703 (2018).

45. P. Bellwood, First Farmers. The Origins of Agricultural Societies, (Wiley-Blackwell, Chichester (2005). 
808 46. D. Baird, et al., Agricultural origins on the Anatolian plateau. Proc. Natl. Acad. Sci. 115, E3077-E3086 (2018).

810 47. A. S. Kassian, et al., Rapid radiation of the inner Indo-European languages: an advanced approach to Indo-European lexicostatistics. Linguistics 59, 949-979 (2021).

812 48. Johannes. Müller Willigen, Samuel van. ,., New radiocarbon evidence for european Bell Beakers and the consequences for the diffusion of the Bell Beaker Phenomenon. Bell Beakers Today 1 (2001).

815 49. R. Bollongino, et al., 2000 years of parallel societies in Stone Age Central Europe. Science 342, 479-481 (2013).

817 50. P. Skoglund, et al., Genomic Diversity and Admixture Differs for Stone-Age Scandinavian Foragers and Farmers. Science 344, 747-750 (2014).

819 51. F. M. T. A. Busing, E. Meijer, R. V. D. Leeden, Delete-m Jackknife for Unequal m. Stat. 820 Comput. 9, 3-8 (1999). 